\title{
Micropruning: The Mechanism of Turning of Aplysia Growth Cones at Substrate Borders in vitro
}

\author{
Donald W. Burmeister and Daniel J. Goldberg \\ Department of Pharmacology and Center for Neurobiology and Behavior, Columbia University College of Physicians and \\ Surgeons, New York, New York 10032
}

\begin{abstract}
Growth cones of Aplysia californica neurons were observed with video-enhanced contrast-differential interference contrast (VEC-DIC) microscopy as they turned at a border between poly-L-lysine-treated and untreated glass. Growth cones that turned generally developed 2 distinct active areas of filopodial and veil formation, much in the way of growth cones undergoing branching. Both active areas advanced, but turning of the neurite occurred through the selective resorption of the incipient branches developing on the untreated substrate. Thus, micropruning of developing regions of the growth cone, rather than the asymmetric extension of filopodia or veils, was primarily responsible for directing neurite growth. We present the hypothesis that abrupt turns by growing neurites are mediated by 2 sets of signals, one causing growth cone splitting, and a second set regulating the survival of the separate branches.
\end{abstract}

The tips of growing axons, the growth cones, often follow intricate pathways through the body to reach their target cells. Growth of many neurites occurs both along straight, relatively decisionless, pathways and through regions, "decision points," where the growth cones must choose from several alternative paths, often abruptly altering their course to do so (Raper et al., 1983a; Landmesser, 1984; Iosney and Landmesser, 1985; Caudy and Bentley, 1986; Bovolenta and Mason, 1987). Correct pathway sclection is an essential step in the formation of proper synaptic connections, and therefore the nature of the signals at decision points and the mechanics of translating the signals into a properly directed axon are major issues in the understanding of neural development.

The chemical composition of the surface on which neurites advance is widely thought to be an important factor in directing neurite outgrowth. Evidence for substrate pathways in vivo arises from the demonstrated preference of particular growth cones, even if experimentally displaced, for particular pathways through the body (Lo and Levine, 1980; Katz and Lasek, 1981). This is most clearly demonstrated in the grasshopper, where identified growth cones show a near-absolute preference for advancing on particular sets of axons (Raper et al., 1983b). Growth cones in

\footnotetext{
Received June 16, 1987; revised Nov. 12, 1987; accepted Jan. 7, 1988.

We wish to thank Mrs. AnnMarie Guzmán for her excellent technical support. This research was funded by NIH Research Grant NS-14711 and by grants from the Stifel Paralysis Research Foundation and the William J. Matheson Foundation. Correspondence should be addressed to Dr. Burmeister, Dept. of Pharmacology, Columbia University College of Physicians and Surgeons, 630 W. 168th St., New York, NY 10032.

Copyright (C 1988 Society for Neuroscience $0270-6474 / 88 / 093151-09 \$ 02.00 / 0$
}

vitro have frequently been shown to discriminate between substrates, in many cases growing on one substrate in preference to another, even though attachment and growth can occur on either when no choice, or a different choice, is offered (Harrison, 1914; Letourneau, 1975b; Bonhoeffer and Huf, 1982; Collins and Lee, 1984; Gundersen and Park, 1984).

One model that could explain this substrate preference is based on the differential adhesion of filopodia (Letourneau, 1981; Bray, 1982; Raper et al., 1983c; Bunge, 1986). In this model, filopodia cmerge from the growth conc, adhere to the substrate, and then exert tension, pulling the growth cone forward. Filopodia falling on the less adhesive of 2 bordering substrates would tend to lose their attachments and be pulled back into the growth cone, while the filopodia on the more adhesive substrate are retained. As a result of this filopodial tug-of-war the growth cone would be pulled onto the more adhesive substrate. Recent work from several laboratories, however, has suggested that filopodial adhesion and the subsequent generation of tension may not be primarily responsible for the advance of the growth cone (Marsh and Letourneau, 1984; Caudy and Bentley, 1986; Goldberg and Burmeister, 1986; Van Zandt and Argiro, 1986; Cheng and Reese, 1987; Aletta and Greene, 1988; but see Bray, 1987) and thus would not in themselves lead to turning. The preferential growth of neurites on a less adhesive substrate has also recently been observed (Gundersen, 1987).

Our own conclusions on the role of filopodia arise from observations of growth cones of Aplysia californica in culture using video-enhanced contrast-differential interference contrast (VECDIC) microscopy (Goldberg and Burmeister, 1986). This technique allows a clear view of changes during axonal growth of both the filopodia and the organellar and plasmalemmal elements of living growth cones. We observed that axon advance always occurs through the same sequence of events: filopodia support the formation of thin, vesicle-free veils on the periphery of the growth cone; the veils fill first with small vesicles, and then with the larger organelles of the central growth cone. The veils are thus transformed, in place, into the new growth cone center. The spread, amorphous morphology of the central growth cone is in turn transformed into the highly polarized structure of the axon cylinder. Axon elongation, therefore, occurs through the extension of a simple structure, the veil, and its subsequent maturation into the axon. The filopodia function primarily as the framework on which veil advance occurs, and can perform this function without firm attachments. Though adhesion of the growth cone is required for the continued maturation of the veil, we saw no evidence that substrate-attached filopodia were pulling forward either the veils or the body of the growth cone.

While we observed that all axon advance occurred through 
the same sequence, the sequence did not always run to completion; not all filopodia supported veil advance, not all the veils matured, and not all the regions that became central growth cone-like in character developed into axon. Turning of the neurite could result, therefore, from the failure of any of these transformations on one side of the growth cone. We were interested, therefore, in observing the behavior of growth cones with VEC-DIC microscopy as they approached and turned at an identifiable "decision point," the border between 2 substrates, first to determine whether the model of differential filopodial adhesion was applicable, and, second, to see whether the bordcr blocked the maturation of veil into neurite at a particular stage. By observing the mechanics of neurite turning in detail, we would then be better able to pinpoint the signaling mechanisms that bring about proper neurite growth.

\section{Materials and Methods}

Substrate preparation. All cells were cultured in $16 \times 32 \times 0.5 \mathrm{~mm}$ wells made by gluing glass coverslips under holes made in plastic petri dishes. A straight, dashed line was scored across the inside surface of the coverslip about midway along the long axis of the well. The dish was inclined and the bottom half of the well filled with $1 \mathrm{mg} / \mathrm{ml}$ polyL-lysine (Sigma; $M_{\mathrm{r}} \approx 400,000$ ) in $0.2 \mathrm{M}$ borate buffer, $\mathrm{pH} 8.2$. The border area was carefully "painted" between the scored lines to form a straight boundary. The scored lines served both as a barrier to the advance of the poly-L-lysine and as a marker for the borderline, which was otherwise invisible under the dissection microscope. The poly-Llysine was left in place overnight without moving the culture dish, and then the coated side was rinsed 5 times with water, followed by several rinses of the entire dish with water. The dishes were used within hours of rinsing.

Cell culture. The dissection and culture of individual neurons from the buccal ganglion of Aplysia californica was identical to that reported previously (Goldberg and Burmeister, 1986). B1 and B2 neurons, but occasionally other large neurons as well, were isolated with microelectrodes and with capillary tubes placed within $1 \mathrm{~mm}$ of the border between treated and untreated glass. The cells were placed between the ends of the scored lines so that the lines did not interfere with either the growth of the axons or with the microscope optics. Culture medium consisted of $50 \%$ L-15 supplemented with salts, to simulate seawater, and 50\% Aplysia hemolymph (Schacher and Proshansky, 1983), or, in one instance, consisted of $100 \%$ modified L- 15 on substrates precoated with hemolymph for $3 \mathrm{hr}$ and rinsed with L-15 immediately prior to culturing. Cells were allowed to grow for $18-36 \mathrm{hr}$ at room temperature before observations were made.

Microscopy. VEC-DIC microscopy was as before (Goldberg and Burmeister, 1986). In order to lessen the exposure of the growth cones to the possible deleterious effects of prolonged bright light, cells were illuminated and viewed for intervals of from 15 to $60 \mathrm{sec}$ (mean, $42 \mathrm{sec}$ ) every 3-15 min (mean, $9 \mathrm{~min} 40 \mathrm{sec}$ ). All observations were recorded on videotape. The video micrographs were prepared from videotaped frames digitized with a $512 \times 480 \times 8$-bit frame grabber (DT2851 with DT2858 coprocessor; Data Translation, Marlboro, MA), with background subtraction and contrast enhancement performed with Data Translation's "Image-Pro" image-processing software.

Morphometry. The various measures of the growth cone were made from repeated viewings of the videotapes. To be included in the tallies, each growth cone had to be (1) actively growing at the beginning of the observations, (2) growing under conditions in which there was an unambiguous borderline visible with VEC-DIC microscopy, (3) in a culture where other neurites were seen to have turned at the border, and (4) under observation for a sufficient amount of time to determine its behavior (generally for more than $30 \mathrm{~min}$; average observation time was $3 \mathrm{hr} 38 \mathrm{~min}$ ). Although growth cones completely free of contact with other growth cones were sought, most did touch other growth cones or neurites while under observation. The veils and filopodia were counted, however, only in observations in which there was no obscuring overlap. The angle of approach of the growth cone to the borderline and the degree of turn were measured at the beginning and end of each observation sequence, using the aligned elements in the proximal region of the growth cone. The distances from the border for newly forming active regions of the growth cones were measured using the Image-Pro imageprocessing system on digitized frames.

For growth cones approaching the border from the poly-L-lysine side, the presence of filopodia, veils, veils filled with small vesicles (filled veils), the central growth cone (defined by the presence of large, irregular refractile bodies, LIRBs, and mitochondria), or the matured neurite on the glass side of the border was noted. The growth cones were frequently observed to split into 2 distinct areas of filopodia and veil formation, separated by a quiescent region; the degree of development of each active area was separately scored. The filopodia and veils of each growth cone were counted and classified according to the substrate they were contacting. Classification of structures crossing the border could be based on either the substrate contacted by the base or on the substrate contacted by the distal portion. Data were analyzed both ways with essentially the same outcome; data based on the distal contact of the structure are presented. For comparisons of growth cones before, during, and after a turning sequence, the start of the turn was defined as the formation of the veil that would eventually mature into the turned neurite. For this measure, splitting growth cones were considered as one until active regions were separated by a region of matured neurite.

\section{Results}

Neurite outgrowth from cultured buccal ganglion neurons plated on glass treated with poly-L-lysine was rapid and vigorous, with a large corona of neurites growing extensively from the cut axon, beginning a few hours after plating and reaching 200-300 $\mu \mathrm{m}$ from the axon by $18-36 \mathrm{hr}$ (Fig. 1A). Neurites described gentle curves with occasional branches that deviated, on average, $37^{\circ}$ $\left(+12^{\circ} \mathrm{SD} ; n=80\right)$ from the parent fiber.

When the cell body and axon were placed on poly-L-lysinetreated areas of glass coverslips and allowed to grow towards a border of untreated glass, a clear demarcation of the borderline was made (Fig. 1A). Axons turned and grew along the borderline, often forming thick fascicles. Few growth cones extended significantly beyond the border region at any time. This was not, however, because the untreated glass could not support growth. Cells plated on untreated glass often showed vigorous growth, with no strong effect of the border on the growth of neurites onto the treated side (Fig. 1B). Growth cones growing on untreated glass were not notably different from growth cones growing on poly-L-lysine-treated glass in our morphometric assays. Using only B1/B2 neurons, we counted filopodia and veils of 44 growth concs in 647 obscrvations, and found no significant differences between growth cones on the 2 substrates in the number of filopodia, veils, or in the ratio of veils to filopodia (Table 1). Growth cones were frequently observed directly straddling the borderline, with their fronts spread onto both substrates (Fig. 2). These growth cones had as many, if not more, filopodia and veils on the untreated as on the treated side (Table 1).

Our quantitative analysis of turning at the border was based on observations of 37 growth cones as they approached or advanced along the border between the treated and untreated glass, growing from the treated side. Sixteen were observed to make significant turns, deviating $45^{\circ}\left( \pm 16^{\circ} \mathrm{SD}\right)$ from their original paths to continue growth on the treated substrate. Of the 21 growth cones that did not turn (deviating only $4^{\circ} \pm 4^{\circ} \mathrm{SD}$ from their original paths), 11 stopped growing after they had crossed the border (8 subsequently undergoing significant retractions), 7 were growing roughly parallel to the borderline in contact with both the treated and untreated substrates throughout the observation period, and 3 were observed to cross the border and continue to advance on untreated glass. Because we did not observe large numbers of fibers growing on untreated glass, and these 3 were not observed for as long as other growth cones, we 

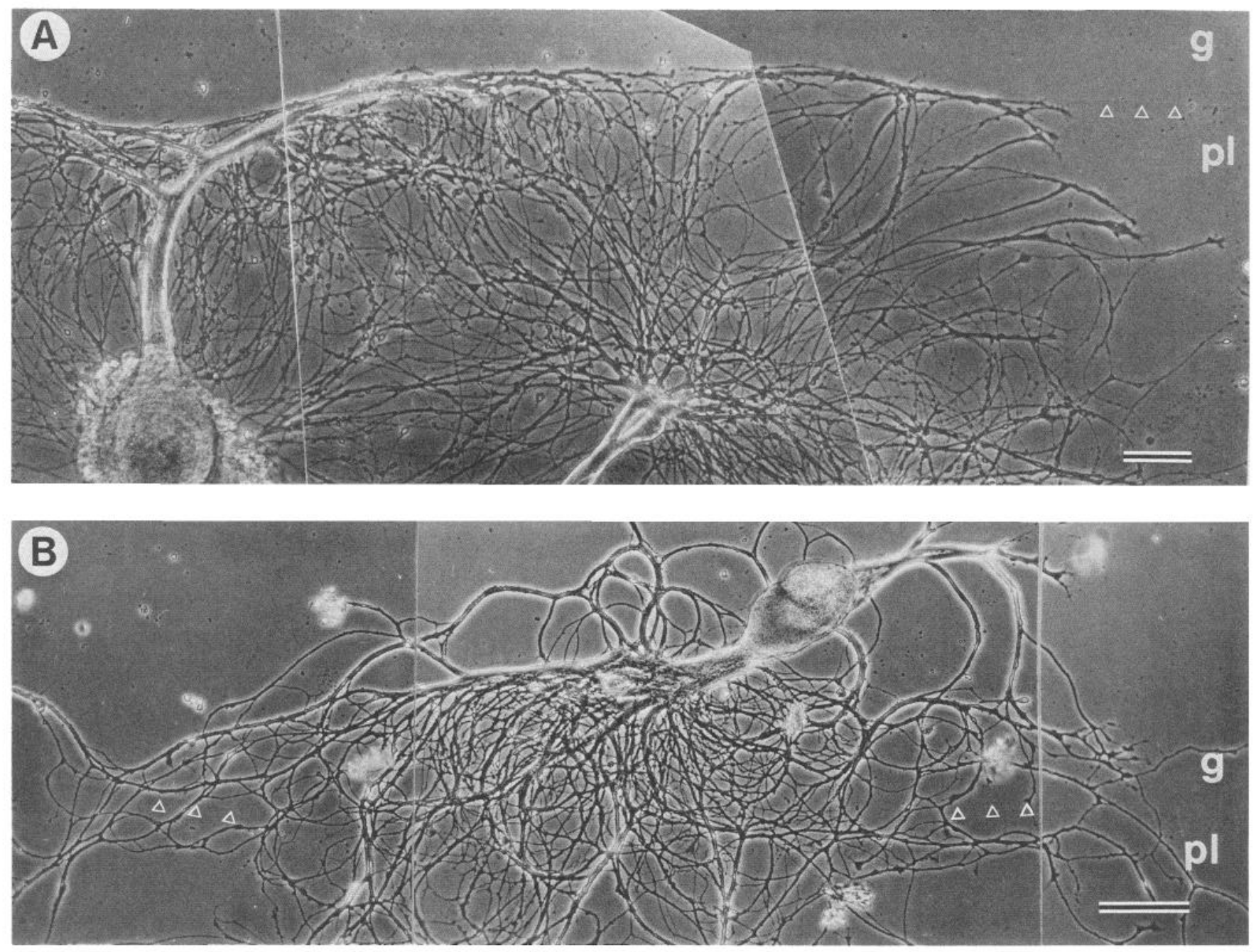

Figure 1. Neurite outgrowth from buccal neurons placed on $(A)$ poly-L-lysine-treated $(p l)$ and $(B)$ untreated $(g)$ glass. Neurites turn and grow along the border between the 2 substrates (triangles) when growing from the treated side, but cross easily without deflection when growing from the untreated side. Bar, $100 \mu \mathrm{m}$.

feel that they too may have eventually stopped growing (see below). The average angle of approach for the 11 growth cones that stopped $\left(55^{\circ} \pm 29^{\circ} \mathrm{SD}\right)$ was significantly larger than the average angle of approach for the 16 that turned $\left(21^{\circ} \pm 19^{\circ} \mathrm{SD}\right.$; Student's $t$ test; $p<0.01$ ). However, among the 16 growth cones that did turn, the degree of turning was not correlated with the angle of approach to the border (correlation coefficient, $R=$ 0.33 ). Rather, the distribution of turning angles at the border resembled the distribution of branch angles at nonborder regions of the treated substrate (Fig. 3).

Turning growth cones regularly developed significant persistent structures on the untreated substrate. We observed 16 growth

Table 1. Growth cones with central regions on untreated substrate or on the borderline

\begin{tabular}{|c|c|c|c|c|}
\hline & \multicolumn{2}{|c|}{ Growth cone substrate } & \multicolumn{2}{|c|}{ Straddling growth cones } \\
\hline & Treated & Untreated & Treated side & Untreated side \\
\hline Filopodia & $12.5 \pm 1.4$ & $14.1 \pm 1.7$ & $7.9 \pm 1.0$ & $8.8 \pm 0.8$ \\
\hline Veils & $2.5 \pm 0.3$ & $2.9 \pm 0.4$ & $1.4 \pm 0.3$ & $2.0 \pm 0.3$ \\
\hline Veils/filopodia & $0.23 \pm 0.02$ & $0.21 \pm 0.03$ & $0.12 \pm 0.02$ & $0.18 \pm 0.02$ \\
\hline$N$ & 32 & 19 & \multicolumn{2}{|c|}{76 (9 growth cones) } \\
\hline
\end{tabular}

Growth cones that had central regions on the untreated substrate or on the borderline did not have a significantly different number of filopodia, veils, or a different ratio of veils to filopodia than did growth cones on the treated substrate (unpaired Student's $t$ tests; $p>0.05$ ). $N$ represents the number of growth cones counted in each condition; 44 growth cones overall were observed, including 7 growth cones observed on both substrates. Multiple observations of one growth cone were averaged. Growth cones that straddled the border, with their forward regions on both substrates, tended to have more filopodia and veils on the untreated side, but only the veil : filopodia ratio was significantly higher (paired Student's $t$ test; $p<0.02$ ). $N$ in this case represents the total number of observations made on 9 growth cones. 


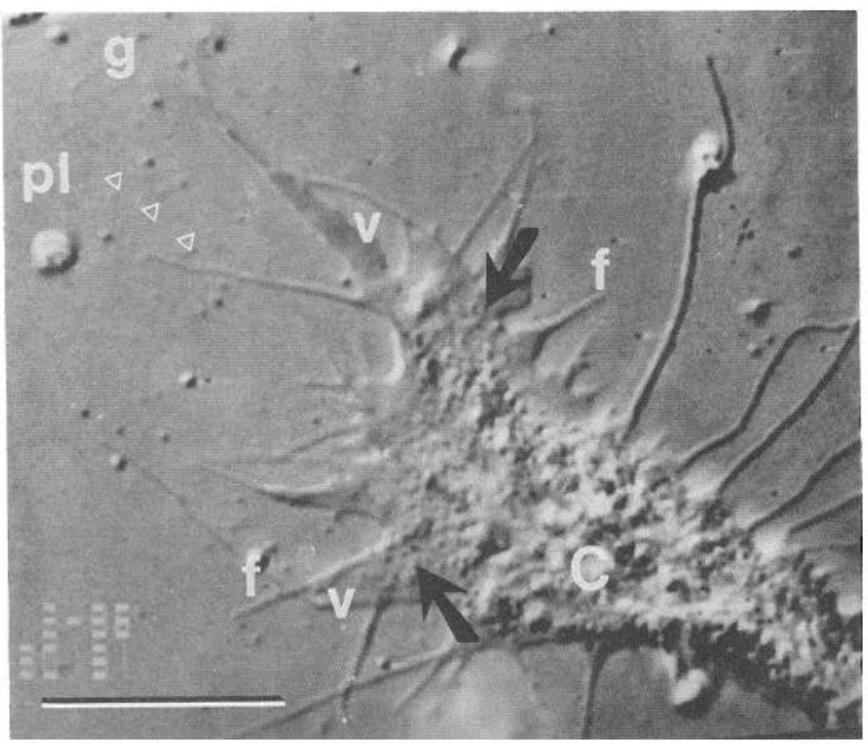

Figure 2. VEC-DIC image of a growth cone straddling the border (triangles) between poly-L-lysine-treated $(p l)$ and untreated $(g)$ glass. Filopodia $(f)$, veils $(v)$, filled veils (arrows), and central growth cone $(C)$ are present on both sides of the border. This growth cone was observed to grow along the border for more than $2 \mathrm{hr}$. Bar, $10 \mu \mathrm{m}$.

cones that turned at the border; all 16 had filopodia firmly attached to the untreated substrate. Fourteen of the 16 cones had veils on the untreated side; in 13 cones, veils filled with small vesicles were on the glass substrate; and in 10 of the cases, the central region of the growth cone, containing mitochondria and LIRBs, was observed to cross over. Matured neurite, containing predominantly aligned elements and supporting directed organelle transport, developed across the border in 5 of the 16 turning sequences.

Figures 4 and 5 illustrate the behavior of several growth cones at the borderline. All 3 growth cones demonstrate the attachment of filopodia and the subsequent development of substantial structures across the border. In Figure 4, a growth cone advances across the border, but a second area of the cone develops and extends along the border. Both active areas show intermittent development and retraction, but the area that maintains contact with the treated glass continues to advance, while the area directed onto the untreated glass withers back to a point several micrometers proximal to the borderline, although filopodial adhesions remain. In Figure 5, 2 growth cones advance across the border. The one approaching at the more acute angle develops 2 distinct branches. After several hours, however, the branch directed across the border begins to show signs of degeneration, and eventually regresses back to where it separated

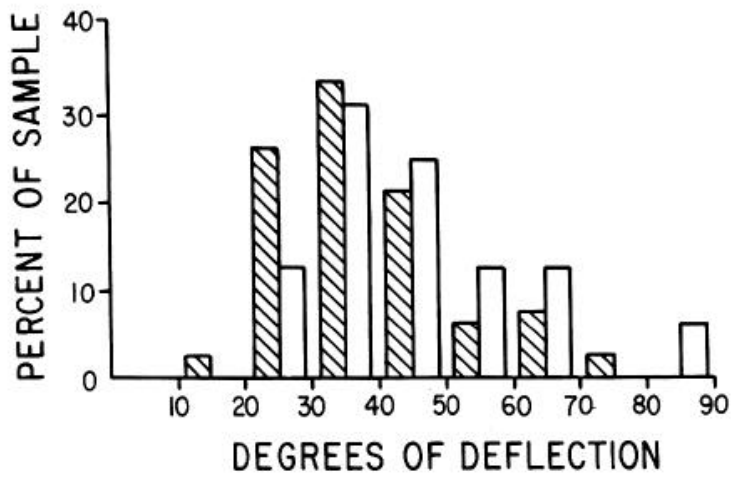

Figure 3. Distribution of branch angles within the axon arborization of $\mathrm{B} 1$ and $\mathrm{B} 2$ neurons growing on treated glass (striped bars; $n=80$ branches from 4 cell arborizations) and distribution of turning angles of neurites at the borders of treated and untreated glass (open bars; $n$ $=16$ growth cones). Branch angles within the arborization were measured as the degree of deflection of the individual branches from the axis of the parent neurite.

from its sister branch, now a point located more than $20 \mu \mathrm{m}$ from the border. The growth cone advancing at an angle more perpendicular to the border, which did not form branches, has meanwhile regressed back to a previous branching point.

The splitting of the growth cone into 2 active regions and the subsequent retraction of developed areas, illustrated in Figures 4 and 5 , occurred in 14 of the 16 turning growth cones we observed. Branching generally occurred close to the border. The first veils of the newly diverging active areas generally formed within $5 \mu \mathrm{m}$ of the border (mean, $5 \pm 6 \mu \mathrm{m} \mathrm{SD}$ ), although in a few large growth cones they arose as far as $17 \mu \mathrm{m}$ from the border (Fig. 6). Active areas directed towards the untreated substrate usually developed substantially before being retracted. We separately scored the development of the active areas (as opposed to the growth cone as a whole) and found that in all but one of the 14 cases where the growth cone split, veils of the active area on the untreated substrate matured to the point of containing small vesicles, and in 5 of the 14 cases the active area developed central growth cone characteristics. The extent of retraction of the active areas was not determined by the substrate border; resorption extended back to the splitting point, regardless of the substrate position (Figs. 4, 5). Because of this and because splitting did not always occur directly over the border, we observed some turned neurites that extended across the border for a distance, as well as neurites that had turned at the border but were substantially displaced from the borderline. The 2 growth cones that turned but did not split were straddling the borderline at the start of the observations, and turned by

\footnotetext{
Figure 4. Turning behavior of a small neurite approaching the border (triangles) between treated $(p l)$ and untreated $(g)$ glass. $a$, The neurite has extended several filopodia across the border region onto the untreated glass; there has been some veil advance in this region as well. Previous observation of this growth cone established that these filopodia were the first to make contact with the untreated substrate (time, $0: 00$ ). $b$, Growth cone continues to advance onto the untreated substrate, including large clumps of material in the central growth cone. Arrow, a distinctive group of filopodia that serves as a landmark in subsequent frames. $f$, A single filopodium at the site of the formation of a second active area (time, 1:15). $c$. A new, actively growing region of the growth cone has emerged $(a 2)$ and grown along the border. The original area of the growth cone that extended across the border has shown some retraction, but still retains filopodial and veil contact with the substrate. Note that nearly all the filopodia of the growth cone are directed towards, and are adherent to, the untreated substrate (time, 1:48). $d$, Area al shows continued viability and continues to show signs of maturation (time, 2:30). $e$. Area a1 begins to regress, while area $\mathrm{a} 2$ has developed into mature neurite with a growth cone located out of the frame (time, 3:32). $f$, Regression of area al has continued and it now extends well back from the substrate border. The
} 

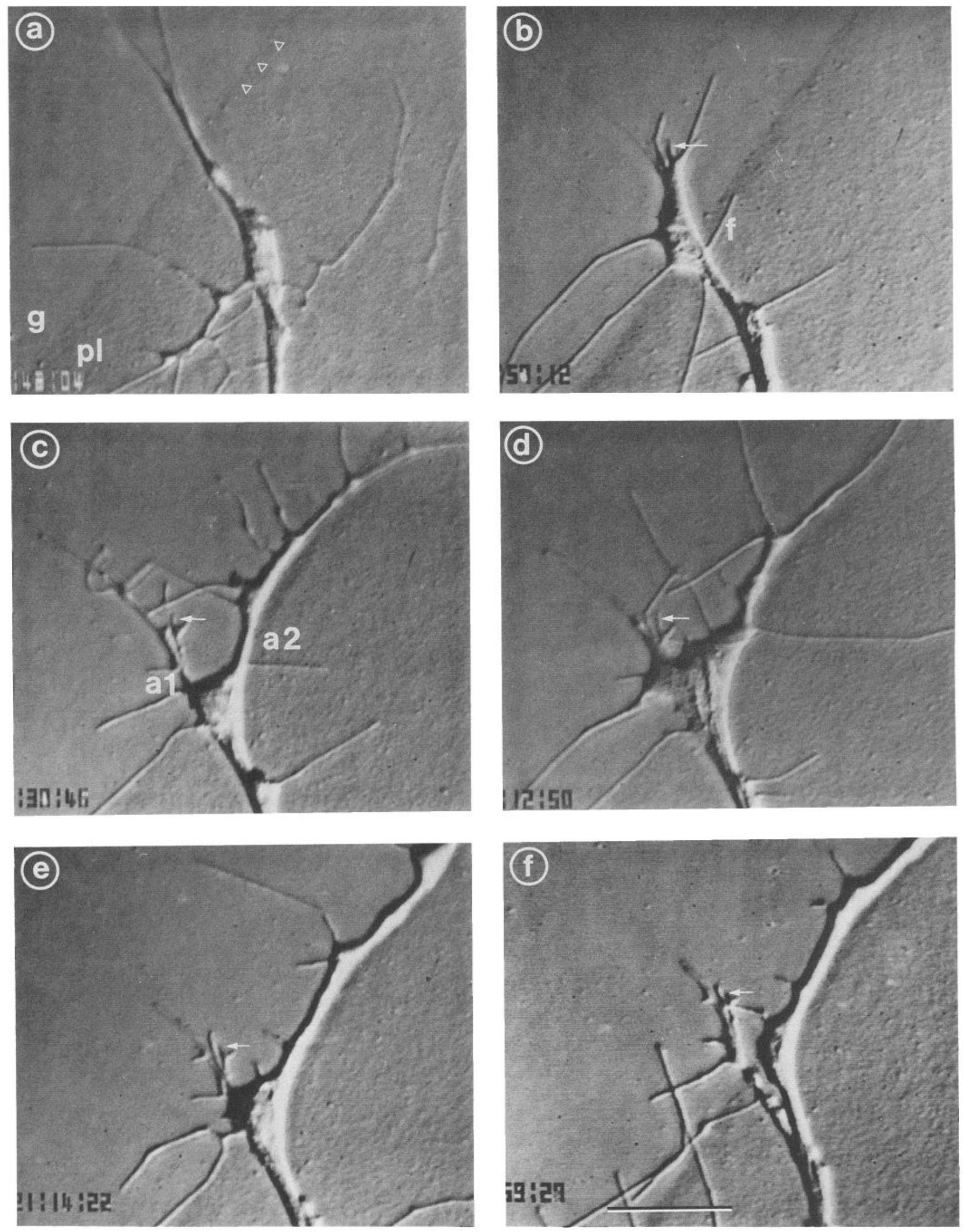

beaded appearance of the regressed neurite is characteristic of these degenerating areas, as shown in Figure 5. Note that several of the filopodial contacts made hours previously remain in place (arrow) (time, 5:17). Bar, $10 \mu \mathrm{m}$. 

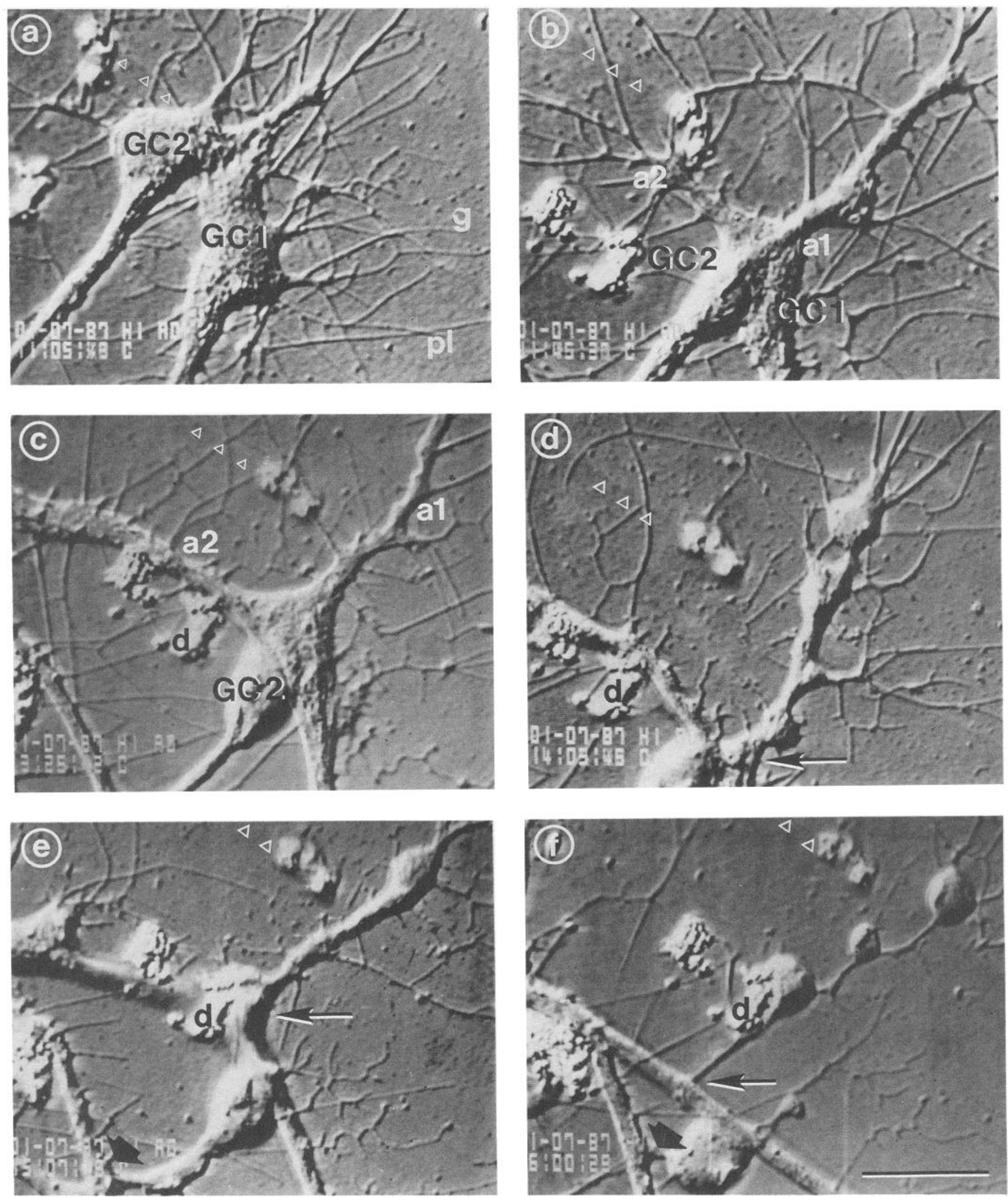

Figure 5. The behavior of 2 growth cones (GC1 and GC2) at the border (triangles) between treated ( $p l)$ and untreated $(g)$ glass. $a$, Both $G C 1$ and $G C 2$ are neurites from the same cell approaching the border from the treated substrate. Note that both growth cones have numerous filopodia that appear adherent to the untreated substrate (time, 0:00). $b$, Both growth cones show the development of matured structures on the untreated substrate. In this frame, the advance of $\mathrm{GCl}$ is seen more clearly. At this time, $\mathrm{GC} 2$ has begun to develop in 2 regions, $a 1$, just beyond the border, and $a 2$, on the treated substrate. The development of these areas is clearer with through-focusing and in the later frames. The overlapping of growth cones with one another and with neighboring neurites was frequently observed (time, 0:35). $c$, Regression of GC2 from the border reveals the full extent of the development of the 2 active areas of GC1. A dirt speck $(d)$ is used as a reference mark in subsequent frames (time, 2:30). $d$, Area al begins 


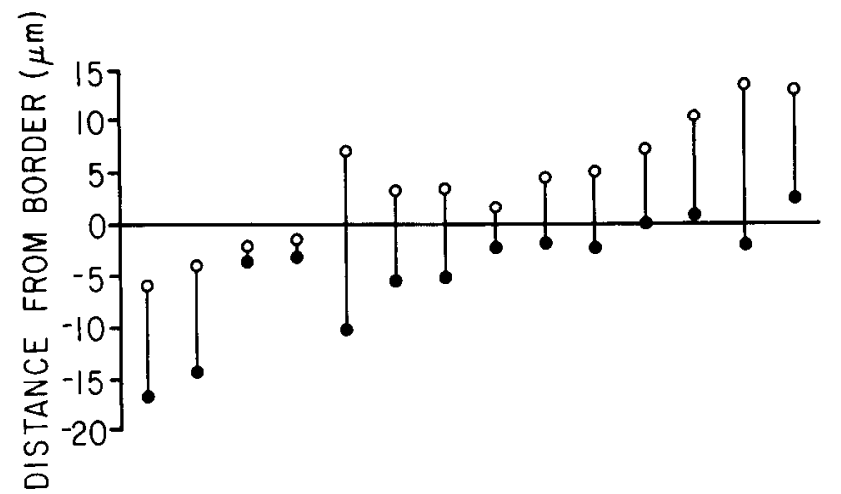

Figure 6. Distance of the 2 active regions of the growth cones from the borderline at the time of the formation of the veil that would eventually become the turned neurite. The distance of the veil growing towards the preferred substrate $(\bullet)$ was measured at the first observation of its formation. At that time, the distance from the border of the most distal veil in the other active area (o) was also measured. Both veils were measured from the center of the leading front of the veil. Because of the complex geometry of the growth cones, the line between the 2 points reflects both the width of the individual growth cone, its angle of approach to the border, and the directions and degrees of development of each active area.

developing veils on the treated surface without any noticeable advance directed onto the untreated substrate.

The numbers of filopodia and veils on the treated and untreated substrates during turning did not dictate the direction of the growth cone turning. There were no differences in the overall number of filopodia, veils, or in the veil : filopodia ratio before, during, or after the start of the turn (as measured on the entire growth cone, i.e., including both active areas). As the growth cones approached the borderline, a high percentage of both veils and filopodia was found on the untreated substrate, so that at the time of the initiation of the turn, only $42 \%$ of the filopodia were found on the treated substrate (Fig. 7). Progressively more filopodia were seen on the treated side as the active area on that substrate advanced.

\section{Discussion}

Turning is caused by asymmetric regression

Turning of growth cones at the border between the poly-L-lysinetreated and untreated glass was attributable mainly to asymmetric regression of developed areas of the growth cone, rather than to asymmetric extension. The untreated substrate did not prohibit the advance or attachment of filopodia or veils nor the subsequent maturation of the growth cone into axon. All the stages of axon formation occurred to some extent in parts of the growth cones extending across the border, but these areas eventually withered in favor of areas or branches that were directed toward the treated side.

Our observations did not suggest that filopodia were directly involved in pulling the growth cone and neurite onto the preferred substrate. Although the asymmetric adhesion of filopodia

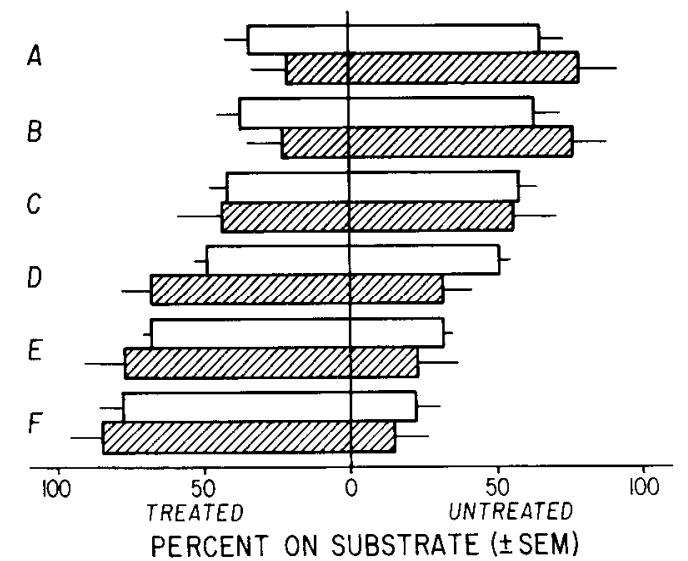

Figure 7. Average $( \pm \mathrm{SEM})$ distribution of filopodia (open bars) and veils (striped bars) of 8 turning growth cones on the treated and untreated substrate as the growth cones approached and turned at the substrate border. The absolute number of filopodia averaged 15.2 \pm 2.4 SEM, and veils, $2.3 \pm 0.6$, and did not vary significantly throughout the turning sequence. Counts represent the total filopodia and veils on the entire growth cone, including all active areas. Multiple observations for each growth cone were averaged within each bin. $A$, More than $20 \mathrm{~min}$ before the initiation of the turn. $B$, At $-20: 00$ to $-0: 01 \mathrm{~min}$. $C$, Time of formation of the veil that would eventually become the turned neurite (time, 0:00). $D$, At 0:01-20:00 min after initiation. $E$, At 20:01-40:00 min after initiation. $F$, More than $40 \mathrm{~min}$ after initiation. Note that as the growth cones approached the border and initiated a turn, a majority of filopodia and veils were directed onto the untreated substrate. As the active region of the growth cone began to advance on the treated side, more veils, then more filopodia, were located on the treated substrate.

at substrate boundaries has frequently been proposed as a mechanism for growth cone turning (Bray, 1987), our study, we believe, is the first to have actually counted filopodia at the boundary. We found that, because the growth cones initially tended to grow beyond the borderline, there were usually more filopodia on the unfavored substrate than on the favored when turning began. It was only as the areas of the growth cone on the favored substrate advanced that the balance of filopodia shifted. The filopodia did not appear to be adhering preferentially to the treated substrate nor to be losing their contacts with the untreated surface as the growth cone turned, and growth cones that straddled the border retained equal numbers of filopodia and veils on both substrates.

In several respects, the behavior of turning growth cones at the substrate border was similar to the behavior of growth cones undergoing branching. Branching growth cones often enlarge and form 2 active regions, which then devclop into scparate branches (Bray, 1973; Wessells and Nuttall, 1978; Goldberg and Burmeister, 1986, Fig. 8; Letourneau et al., 1986). Similarly, when the growth cones here were in the vicinity of the substrate boundary, 2 regions of the growth cone became particularly active in the formation of veils and filopodia, generally developing as 2 incipient branches. Neurite branches have characteristic angles, depending on both the cell type and the substrate

to vesiculate, but continues to maintain contacts with the substrate. The regression extends beyond the borderline to the point of intersection with branch a1 (arrow) (time, 3:00). e, After showing severe signs of degeneration, area a l seems to lose all internal integrity and slide up the neurite (arrow); note the straightened appearance of $\mathrm{a} 1$ and the positions of the branches relative to dirt speck $d$. Despite this movement, several filopodial attachments to the substrate remain. The broad arrow indicates the branching point from which $\mathrm{GCl}$ extended towards the border (time, 4:02). $f$, Branch al loses almost all its connection to the neurite (arrow indicates narrow remaining connection) but maintains its adherence to both the treated and untreated substrates. GC1 has also been nearly completely resorbed (time, 5:55). Bar, $10 \mu \mathrm{m}$. 
conditions (Bray, 1973; Kat7, 1985). We found that the angle of deflection of turning neurites at the border was nearly the same as the angle of deflection of branches in the axonal arbor at nonborder regions. Interestingly, the turning angle was not correlated with the angle of approach to the border, indicating that internal constraints may have determined the degree of turn.

The splitting of growth cones generally occurred close to the borderline, but we do not know whether the splitting was induced by the border or whether the borderline accentuated a natural tendency to branch. Little is known about the causes of neurite branching. Growth cone splitting occurs when advancing neurites encounter a physical obstacle (Speidel, 1933) or when forwardly directed filopodia are physically disrupted (Wessells and Nuttall, 1978). Highly adhcsive substrates increase the frequency of growth cone splitting (Letourneau, 1975a; Bray et al., 1987), while taxol, a drug that promotes microtubule polymerization, reduces it (Letourneau et al., 1986). Finally, in neurites in which the axon is not well adherent to the substrate, and thus exerts tension on the growth cone, cutting the axon results in splitting (Bray, 1979). None of these studies immediately explains how a substrate boundary might induce splitting. The substrate border we visualized may have been a region with a higher concentration of poly-L-lysine than other areas of the substrate (Gundersen, 1987); however this was not in itself sufficient to induce branching of straddling growth cones nor of growth cones growing from the untreated substrate. Thus, even though 14 of 16 cases of borderline turning involved the formation of a split growth cone, and most splits occurred within $5 \mu \mathrm{m}$ of the border, we can only conjecture that the border acted as a trigger for branching.

The major difference between branching on a homogeneous substrate and branching at the substrate border was that, at the border, only one of the incipient branches survived. By splitting at the border, the growth cones created 2 active areas with unequal contact with the preferred substrate; thus a competition between the incipient branches resulted, leading to the micropruning of the unfavored area. Regression from the border did not only occur in splitting growth cones, however. An observation that initially puzzled us was that neurites that approached the border at near-perpendicular angles did not turn; most grew across the border, stopped, and then retracted. Because neurites do not usually branch at sharp angles from the parent fiber, perhaps because of internal cytoskeletal constraints (Katz, 1985; Letourneau et al., 1986), these growth cones were probably not able to direct branches onto the favorable substrate. They now reacted, however, in the same way as the unfavored incipient branch of a splitting growth cone. The entire growth cone and neurite regressed, often over many micrometers, to the previous branch node. This suggests that the competition between 2 incipient branches on different substrates and the competition between 2 whole growth cones on different substrates could share the same mechanisms; that micropruning of growth cones at the border was similar to the pruning of unsuccessful branches seen in the development of most nervous systems.

What is the mechanism underlying the micropruning of areas of the growth cone or, indeed, the pruning of large branches from the border region? Regression does not appear to occur because of a loss of substrate adhesion. First, when regression occurs, many contacts with the substrate remain, both along filopodia and at areas that underlay the more matured regions of the advancing growth cones. Rather than having been pulled off the surface and resorbed, the projections seem to have degenerated from within, leaving behind an intact membranous sheath. Second, the regressions do not stop at the border, but proceed back to the branch node, despite the presumed adhesive differences at the border. In some respects, the regression of areas extending across the border is similar to the behavior of growth cones, which withdraw when they contact the neurites of other cell types (Kapfhammer and Raper, 1987). [We have never observed the $\mathrm{B} 1$ and $\mathrm{B} 2$ growth cones retracting from contact with similar neurites or growth cones, in agreement with observations of the interactions of other homotypic neurons (Bray et al., 1980; Kapfhammer et al., 1986).] Although the internal changes leading to the withdrawal are not understood, we can draw some conclusions about the nature of the signaling. The untreated glass substrate cannot be thought of as simply a noxious signal, or an inappropriate substrate for growth. Although the survival of cells on untreated glass appears more tenuous than on poly-L-lysine treated glass, many cells plated on untreated glass do produce strong outgrowths, with growth cones that advance in a manner identical to that seen on other substrates. It is also not uncommon to observe growth cones that straddle the border, growing in contact with both substrates, with no obvious reaction on the part of structures in contact with the untreated glass. It is only under conditions in which one of 2 active areas of the growth cone or axonal arbor is on the preferred substrate and the other is on the less preferred substrate that the type of regressive event we observed in turning or retracting growth cones occurred.

We thus feel that competition between branches or areas of the growth cone is central to understanding the turning we observed, but that the controlling mechanism cannot simply enhance the growth of one incipient branch, but must also (or perhaps solely) decrease the viability of the competing branch. One intracellular phenomenon that may prove important is the partitioning of axonally transported material between branches. Recently, we correlated the cessation of growth and a concomitant decrease in the volume of vesicular transport in one branch of a bifurcate axon with the formation of synaptic conncctions by the other branch. In this case, growth and transport are reduced only in the branch that does not contact the target (Schacher, 1985; Goldberg and Schacher, 1987). A similar phenomenon within the growth cone could account for the withering of incipient branches and would explain why the withering extends back to branch nodes.

\section{Are there two sets of signals for turning?}

The sharp turns made by growth cones at a substrate boundary seemed to involve 2 distinct events: first, the formation of 2 separate, active growth areas, and, second, the selective regression of one of them. Although the behavior of growth cones on artificial, 2-dimensional substrates may appear overly simplified, several in vivo studies have demonstrated growth cone behavior that is similar to or consistent with this scheme. In the grasshopper cmbryo, for cxample, developing Til axons with fairly compact growth cones extend along the limb bud until they encounter a boundary region, where they broaden and extend for several branches. When some filopodia make contact with a specific "guidepost" cell, however, growth of the misdirected branches ceases, and they are not evident in the mature animal (Bentley and Caudy, 1983a). Contact with the guidepost cell appears to be a signal for branch pruning, because the branches are retained when the guidepost cell is removed (Bent- 
ley and Caudy, 1983b). There is also cvidence that micropruning occurs in vertebrates. The growth cones at decision points in the lumbosacral plexus of the chick and in the optic chiasma of the rat are larger and have a more irregular form than do the growth cones from the same axons growing along pathways where no directional decisions are made (Tosney and Landmesser, 1985; Bovolenta and Mason, 1987). Advancing neurites in the optic chiasm, for example, have areas that appear to be the remnants of misdirected growth cone extensions (Bovolenta and Mason, 1987).

Dividing the process of pathway selection into 2 activities clarifies the signaling requirements at developmental decision points. The decision point must first initiate the changes in the growth cone that mediate a sharp turn, a process that, we propose, is identical to branching. This signal to branch need not encode specific directional information, however. The decision as to the survival of each branch could be determined secondarily by a wide spectrum of conditions, ranging from local substrate interactions to the formation of synaptic and trophic contacts by one of the branches. Our observations suggest, furthermore, that the micropruning observed at the level of the growth cone and the pruning of neurites of more distantly related neurites may share a common mechanism.

\section{References}

Aletta, J. M., and L. A. Greene (1988) Growth cone configuration and advance: A time-lapse study using video-enhanced differential interference contrast microscopy. J. Neurosci. 8: 1425-1435.

Bentley, D., and M. Caudy (1983a) Navigational substrates for peripheral pioneer growth cones: Limb-axis polarity cues, limb segment boundaries, and guidepost neurons. Cold Spring Harbor Symp. Quant. Biol. 48: 573-585.

Bentley, D., and M. Caudy (1983b) Pioneer axons lose directed growth after selective killing of guidepost cells. Nature 304: 62-65.

Bonhoeffer, F., and J. Huf (1982) In vitro experiments on axon guidance demonstrating an anterior-posterior gradient on the tectum. EMBO J. 1: 427-431.

Bovolenta, P., and C. Mason (1987) Growth cone morphology varies with position in the developing mouse visual pathway from retina to first targets. J. Neurosci. 7: 1447-1460.

Bray, D. (1973) Branching patterns of individual sympathetic neurons in culture. J. Cell Biol. 56: 702-712.

Bray, D. (1979) Mechanical tension produced by nerve cells in tissue culture. J. Cell Sci. 37: 391-410.

Bray, D. (1982) Filopodial contraction and growth cone guidance. In Cell Behavior, R. Bellairs, A. Curtis, and G. Dunn, eds., pp. 299-317, Cambridge U. P., Cambridge, UK.

Bray, D. (1987) Growth cones: Do they pull or are they pushed? Trends Neurosci. 10:431-434.

Bray, D., P. Wood, and R. P. Bunge (1980) Selective fasciculation of nerve fibres in culture. Exp. Cell Res. 130: 241-250.

Bray, D., M. B. Bunge, and K. Chapman (1987) Geometry of isolated sensory neurons in culture. Exp. Cell Res. 168: 127-137.

Bunge, M. B. (1986) The axonal cytoskeleton: Its role in generating and maintaining cell form. Trends Neurosci. 9: 477-482.

Caudy, M., and D. Bentley (1986) Pioneer growth cone steering along a series of neuronal and non-neuronal cues of different affinities. $J$. Neurosci. 6: 1781-1795.

Cheng, T. P. O., and T. S. Reese (1987) Video analysis of growth cone movement during neuritic extension. J. Cell Biol. 105: 128a.

Collins, F., and M. R. Lee (1984) The spatial control of ganglionic neurite growth by the substrate-associated material from conditioned medium: An experimental model of haptotaxis. J. Neurosci. 4: 28232829 .
Goldberg, D. J., and D. W. Burmeister (1986) Stages in axon formation: Observations of Aplysia axons in culture using video-enhanced contrast-differential interference contrast microscopy. J. Cell Biol. 103: 1921-1931.

Goldberg, D. J., and S. Schacher (1987) Differential growth of the branches of a regenerating bifurcate axon is associated with differential axonal transport of organelles. Dev. Biol. 124: 35-40.

Gundersen, R. W. (1987) Response of sensory neurites and growth cones to patterned substrata of laminin and fibronectin in vitro. Dev. Biol. 121: 423-431.

Gundersen, R. W., and K. H. C. Park (1984) The effects of conditioned media on spinal neurites: Substrate-associated changes in neurite direction and adherence. Dev. Biol. 104: 18-27.

Harrison, R. W. (1914) The reaction of embryonic cells to solid structurcs. J. Exp. Zool. 17: 521-544.

Kapfhammer, J. P., and J. A. Raper (1987) Collapse of growth cone structure on contact with specific neurites in culture. J. Neurosci. 7 . 201-212.

Kapfhammer, J. P., B. E. Grunewald, and J. A. Raper (1986) The selective inhibition of growth cone extension by specific neurites in culture. J. Neurosci. 6: 2527-2534.

Katz, M. J. (1985) Axon branch shapes. Brain Res. 361: 70-76.

Katz, M. J., and R. J. Lasek (1981) Substrate pathways demonstrated by transplanted Mauthner axons. J. Comp. Neurol. 195: 627-641.

Landmesser, L. (1984) The development of specific motor pathways in the chick embryo. Trends Neurosci. 7:336-339.

Letourneau, P. C. (1975a) Possible roles for cell-to-substratum adhesion in neuronal morphogenesis. Dev. Biol. 44: 77-91.

Lctourncau, P. C. (1975b) Ccll-to-substratum adhesion and guidance of axonal elongation. Dev. Biol. 44: 92-101.

Letourneau, P. C. (1981) Immunocytochemical evidence for colocalization in neurite growth cones of actin and myosin and their relationship to cell-substratum adhesions. Dev. Biol. 85: 113-122.

Letourneau, P. C., T. A. Shattuck, and A. H. Ressler (1986) Branching of sensory and sympathetic neurites in vitro is inhibited by treatment with taxol. J. Neurosci. 6: 1912-1917.

Lo, R. Y. S., and R. L. Levine (1980) Time course and pattern of optic fiber regeneration following tectal lobe removal in the goldfish. J. Comp. Neurol. 191: 295-314.

Marsh, L., and P. C. Letourneau (1984) Growth of neurites without filopodial or lamellipodial activity in the presence of cytochalasin B. J. Cell Biol. 99: 2041-2047.

Raper, J. A., M. J. Bastiani, and C. S. Goodman (1983a) Pathfinding by neuronal growth cones in grasshopper. I. Divergent choices made by the growth cones of sibling neurons. J. Neurosci. 3: 20-30.

Raper, J. A., M. J. Bastiani, and C. S. Goodman (1983b) Pathfinding by neuronal growth cones in grasshopper. II. Selective fasciculation onto specific axonal pathways. J. Neurosci. 3: 31-41.

Raper, J. A., M. J. Bastiani, and C. S. Goodman (1983c) Guidance of neuronal growth cones: Selective fasciculation in the grasshopper embryo. Cold Spring Harbor Symp. Quant. Biol. 48: 587-598.

Schacher, S. (1985) Differential synapse formation and neurite outgrowth at two branches of the metacerebral cell of Aplysia in dissociated cell culture. J. Neurosci. 5: 2028-2034

Schacher, S., and E. Proshansky (1983) Neurite regeneration by Aplysia neurons in dissociated cell culture: Modulation by Aplysia hemolymph and the presence of the initial axonal segment. J. Neurosci. 3: 2403-2413.

Speidel, C. C. (1933) Studies of living nerves II. Am. J. Anat. 52: 175.

Tosney, K. W., and L. T. Landmesser (1985) Growth cone morphology and trajectory in the lumbosacral region of the chick embryo. J. Neurosci. 5: 2345-2358.

Van Zandt, W., and V. Argiro (1986) Growth cone foot-prints in motion: Three dimensional views by computer-enhanced interference reflection microscopy. Soc. Neurosci. Abstr. 12: 369.

Wessells, N. K., and R. P. Nuttall (1978) Normal branching, induced branching, and steering of cultured parasympathetic motor neurons. Exp. Cell Res. 115: 111-122. 